\title{
A Fatal Case of Cerebral Melioidosis
}

\section{Raina Chaudhary', Alina Singh', Manoj Pradhan ${ }^{1}$, Reeba Karki² and Paawan Bahadur Bhandari ${ }^{3}$}

${ }^{1}$ Department of Microbiology, Nepalese Army Institute of Health Sciences, Shree Birendra Hospital, Chhauni, Kathmandu, Nepal.

${ }^{2}$ Department of ENT, Head \& Neck Surgery, Nepalese Army Institute of Health Sciences, Shree Birendra Hospital, Chhauni, Kathmandu, Nepal.

${ }^{3}$ Department of Neurosurgery, Nepalese Army Institute of Health Sciences, Shree Birendra Hospital, Chhauni, Kathmandu, Nepal.

\section{ABSTRACT}

Introduction: Melioidosis is potentially fatal type of infectious disease caused by soil saprophytes Burkholderia pseudomallei. It is endemic to Southeast Asia and Northern Australia. We report the case of Cerebral Melioidosis which was consequences of acute otitis media. Patient was treated with Ceftazidime and Meropenem, despite of that patient died. To the best of our knowledge, this is the first case of cerebral melioidosis from Nepal.

Key words: Burkholderia pseudomallei; Melioidosis

Correspondence: Raina Chaudhary, Department of Microbiology, Nepalese Army Institute of Health Sciences, Shree Birendra Hospital, Chhauni, Kathmandu, Nepal. Email: raina334004@gmail.com

DOI: http://dx.doi.org/10.3126/mjsbh.v18i2.22390 


\section{INTRODUCTION}

Melioidosis is a potentially fatal illness caused by a soil saprophyte, Burkholderia pseudomallei, a Gram-negative bacterium, with the predominant mode of transmission being percutaneous inoculation. ${ }^{1}$ It causes disease in human $\&$ animal. It is a disease of public health importance in Southeast Asia and Northern Australia. $^{2}$

The first case of Melioidosis was documented in Burma in 1911. Malaysia \& Singapore also reported Burkholderia pseudomallei in 1913 and then Vietnam reported it in 1925. It is known to be highly endemic in northeast Thailand and Northern Australia, where annual incidence rates ranging from 4 to 41.7 cases per 100,000 population. ${ }^{1,2}$ The first case in South Asia was confirmed in Sri Lanka in 1927 and first diagnosed case in India was in 1953. ${ }^{3}$ There are several cases, which has been reported from different parts of India like Kerala, Tamil Nadu, Eastern and North-Eastern part. ${ }^{4}$ However, there is only one reported case from Nepal in the year 2005 which was respiratory melioidosis. ${ }^{5}$ Here, we would like to report a case of cerebral Melioidosis.

\section{CASE REPORT}

A 22 years old male, serving soldier was brought from commando training academy to Tertiary Care Hospital with a history of pus discharge from his right ear for over 10 days associated with pain, high grade fever and decreased hearing with tinnitus. Within 24 hours of admission he started vomiting

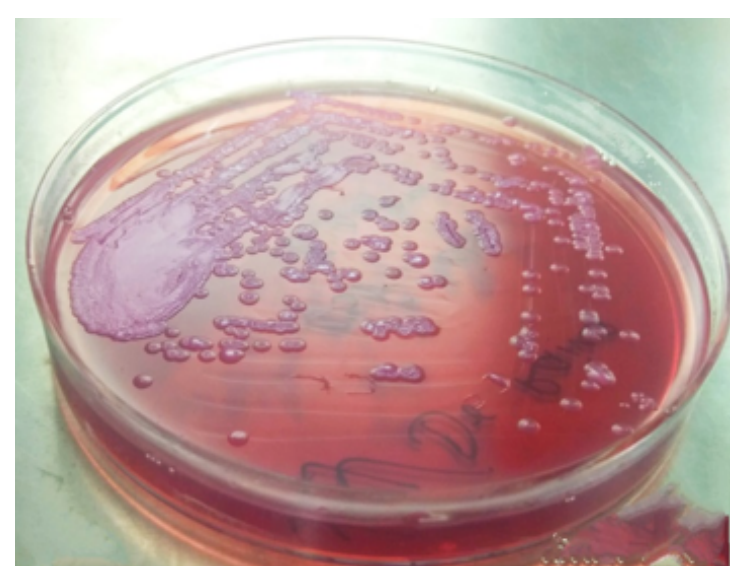

Figure 1. Pinkish rugose colonies with metallic sheen on MacConkey Agar for more than three episodes and became disoriented.

On examination, the patient was febrile, conscious but disoriented and signs of meningeal irritation were positive. Discharge from aural cavity with posterior superior sagging of right ear was observed on local examination. The patient was treated with Vancomycin and Ceftriaxone.

Laboratory results showed elevated inflammatory parameters on complete blood count; Leukocytes: 12,100/cmm, Neutrophil: 80\%. Cerebrospinal fluid (CSF) analysis was suggestive of bacterial meningitis, with total count of $180 / \mathrm{mm}^{3}$ with predominance of polymorphonuclear leukocytes. Biochemical analysis of CSF was also suggestive of bacterial meningitis with glucose: $42 \mathrm{mg} / \mathrm{dl}$ and protein $181 \mathrm{mg} / \mathrm{dl}$. However, rest of the parameters were within normal limit indicating no other organ was involved during disease process. Direct Gram stain did not reveal any organism, but showed plenty of polymorphoneuclear leukocytes suggesting inflammatory process. Acid fast staining was also done, but didn't reveal any acid fast bacilli.

Cerebrospinal fluid was inoculated on BacT/3D Alert System (bioMérieux) colorimetric-based detection system. Within 18 hours, BacT/3D Alert gave alarm for positivity and then specimen was subcultured on Blood Agar, Chocolate Agar and MacConkey Agar. Pus from aural cavity was also collected and inoculated accordingly. Next day,

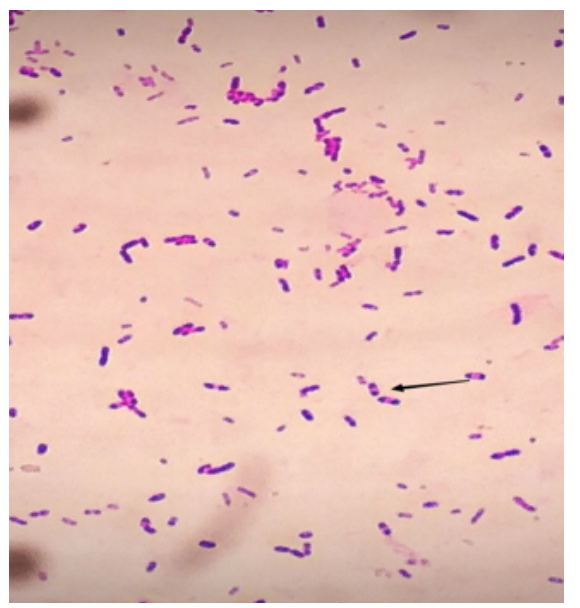

Figure 2. Gram negative bacilli with typical "Safety pin appearance" 


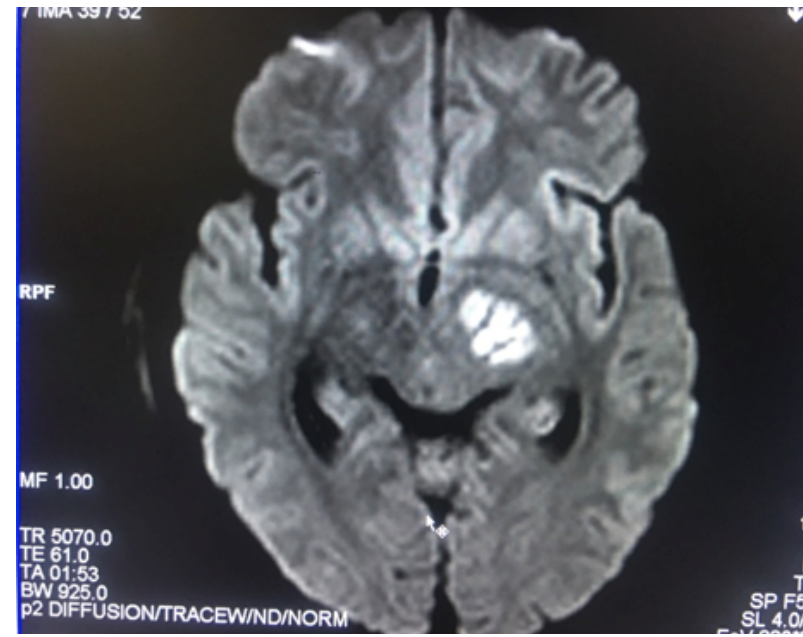

Figure 3. Contrast MRI brain revealed patchy enhancement involving brainstem and left thalamic region

both samples showed dry wrinkled colonies on Blood Agar and pinkish rugose colonies with metallic sheen on MacConkey Agar (Figure 1).

Gram staining of the isolates revealed Gram negative bacilli with typical "Safety pin appearance" (Figure 2). Various biochemical reactions and other cultural characteristics were carried out which showed non-fermenter, catalase and oxidase positive, motile and resistant to Polymyxin B and various commonly used antibiotics like aminoglycosides, macrolides, first and second generation cephalosporin and penicillin. Further confirmation of isolated organism was done by Vitek 2 compact which identified the test organism to be Burkholderia pseudomallei.

Contrast MRI brain revealed patchy enhancement involving brainstem and left thalamic region (Figure 4). On the basis of radiological and microbiological findings, the patient was diagnosed as a case of Cerebral Melioidosis. Interpretative criteria of antibiotic for this organism are not mentioned in recent Clinical Laboratory Standard Institute (CLSI) guideline. Also there are no national guidelines for treating meliodosis in Nepal or other South Asian countries. So we took threshold zone sizes for member of the family Enterobactericeae and Pseudomonas aeruginosa as reported earlier. We performed disc diffusion test which showed isolate sensitive to Ceftazidime, Meropenem, Co-trimoxazole and Amoxycillin+
Clavulanic acid. Therefore, the patient was treated with combination therapy of Meropenem \& Ceftazidime. In many instances there has been treatment failure even in vitro susceptibility was observed for these drugs.

On third day of admission, emergency Modified Radical Mastoidectomy was performed. But unfortunately, patient deteriorated after surgery and was put on artificial ventilation due to low Glasgow Coma Scale. Despite aggressive treatment, the patient's condition continued to deteriorate and finally succumbed on the twelfth day.

\section{DISCUSSION}

Burkholderia pseudomallei is a small, Gramnegative, oxidase-positive, motile, aerobic bacillus with occasional polar flagella. ${ }^{1}$ It has been described as a biothreat agent due to risks of aerosolisation, low infectious dose and high mortality rate even with antimicrobial therapy. ${ }^{6}$

This bacterium was first described in 1911 by Whitemore and Krishnaswami as septicemic disease in morphine addicts in Rangoon, Burma. ${ }^{1}$ It has a specific ecological niche, existing in soil and stagnant water in an area of latitudes 20 degrees North and South of equator, primarily in Thailand, Vietnam and parts of northern Australia. ${ }^{1}$

Acquisition from environment occurs through percutaneous inoculation, ingestion or inhalation. Melioidosis primarily results from contact with contaminated soil or water; person to person transmission is rare. The incubation period ranges from one to 21 days (mean $=9$ days). ${ }^{1}$ However, an incubation period as long as 62 years has been described. ${ }^{10}$ In most endemic regions, there is close association between melioidosis and rainfall. In Thailand and northern Australia, 75\% and 85\% of cases respectively, have occurred in the wet season. ${ }^{1}$

Although widely known as a soil saprophyte, only limited studies has been carried out to isolate Burkholderia pseudomallei from environment. This is very important while establishing melioidosis that has been attributed by environmental factors. The steps involved are very cumbersome and 
requires laborious laboratory work and need for essential lab equipment and enrichment and selective media like Ashdown's media. Molecular techniques like PCR and mass spectrometry are also necessary for conclusive results. A study in Tamil Nadu India confirmed four Burkholderia pseudomallei isolates from rice paddy samples. Similar study in Kerala, India also found $22.7 \%$ of the isolated Burkholderia species to be Burkholderia pseudomallei. ${ }^{7-9}$

Melioidosis is a fascinating type of infection in terms of its pathogenesis. The outcome of the hostpathogen interaction ranges from asymptomatic seroconversion to rapidly fatal overwhelming sepsis. ${ }^{3}$ The potential severity of the disease is described by its potential virulence factors like polysaccharides capsule and lipopolysaccharide. They are responsible for resistant to complement mediated killing. Diabetes, alcohol excess and renal diseases are the major risk factors. ${ }^{2}$

Melioidosis has enormous clinical diversity, spanning asymptomatic infection, localised skin ulcers or abscesses, chronic pneumonia, mimicking tuberculosis and fulminant septic shock with abscesses in multiple internal organs. Therefore it results in diagnostic challenges; at the same time lack of awareness among clinician and lack of resources for identification of bacteria add diagnostic difficulties. ${ }^{10}$

The standard diagnostic method for Burkholderia pseudomallei is culture. However, there is always a high possibility of misidentification of it as a contaminant or another species. In suspected cases of melioidosis, it is always recommended that urine and throat swab is cultured in selective media with simultaneous blood culture for better isolation. For further identification of the species, many commercially available systems such as API 20NE, Phoenix (Becton, Dickinson and Company) and VITEK (BioMieux) can be used. Several other direct diagnostic methods have been developed based on Immunofluorescence Assay (IFA), PCR and Lateral Flow Immunoassay. Among these tests, the Active Melioidosis Detect Lateral Flow immunoassay (AMD-LFA) (InBios Inc., Seattle, WA, USA), has shown promising results as a 'point of care' (POC) test for early diagnosis of melioidosis. ${ }^{11}$

Melioidosis is an emerging health concern in South Asia as its number has been alarmingly increasing in neighbouring countries like India where it was predicted more than 50,000 cases and 30,000 deaths each year. ${ }^{3}$ In Nepal, most of the cases are likely to exist undiagnosed as in other South Asian countries. Diagnostic microbiology services, laboratory based surveillance remains poor, especially those serving the rural poor, which is very group that is at greatest risk of melioidosis. ${ }^{3}$ This calls for need of more researches, effective preventive measures and surveillance system to address and improve knowledge of the disease distribution and burden.

In our case, it was believed that patient might have acquired the organism from environment while doing training. Seasonal factors can also be considered for acquiring disease. His specific predisposing factor was acute otitis media.

\section{CONCLUSIONS}

The main purpose of this case report is to promote awareness about this uncommon infection leading to serious complication like cerebral abscess among clinicians, lab personnel and patients. Because of the severity of this infection, patient should not delay seeking immediate investigation and treatment. Early diagnosis and appropriate treatment is crucial in reducing serious complications leading to high mortality.

To cite this article: Chaudhary R, Singh A, Pradhan M, Karki R, Bhandari PB. A Fatal Case of Cerebral Melioidosis. MJSBH. 2019;18(2):64-8.

Conflict of Interest: None declared 


\section{REFERENCES}

1. Currie B J. Burkholderia pseudomallei, Burkhloderia mallei: Melioidosis and Glanders. In: Mandell GL, Bennett JE, Dolin R, editors. Mandell, Douglas and Bennett's Principle and practice of infectious Disease. 7th ed. Philadelphia: Churchill Livingstone, 2010; 2869-77.

2. Cheng AC, Currie BJ. Melioidosis: epidemiology, pathophysiology and management. Clin Microbiol Rev. 2005;18: 383-416.

DOI: https://doi.org/10.1128/CMR.18.2.383-416.2005

3. Mukhopadhyay C, Shaw T, Varghese GM, Dance DAB. Melioidosis in South Asia (India, Nepal, Pakistan, Bhutan and Afghanistan). Tropical Medicine and Infectious Disease. 2018;3(2):51

DOI: https://doi.org/10.3390/tropicalmed3020051

4. Singh A, Grover N, Gupta S, Bhatt P, Sahni A. Disseminated melioidosis: a case report. Rev in Med Microbiol. 2015; 26(3):116-8.

DOI: https://doi.org/10.1097/MRM.0000000000000034

5. Shrestha NK, Sharma SK, Khanal B, Dhakal SS. Melioidosis imported into Nepal. Inf Dis. 2005;37(1):64-6. DOI: https://doi.org/10.1080/00365540410026040

6. Pelerito A, Nunes A, Coelho S, Piedade C, Paixão P, Cordeiro R, et.al. Burkholderia pseudomallei: First case of melioidosis in Portugal. ID Cases. 2016;3:10-1.

DOI: https://doi.org/10.1016/j.idcr.2016.01.004

7. Njauy V, Lemeshev Y, Sadkowski L, Crawford G. Cutaneous melioidosis in a man who was taken as a prisoner of war by the Japanese during World War II. J Clin Microbiol. 2005;43:970-2.

DOI: https://doi.org/10.1128/JCM.43.2.970-972.2005

8. Chandrakar S, Dias M. Soil Sampling of Burkholderia pseudomallei in and around Mangalore, India - an Explorative Study. J Bacteriol Mycol. 2017;4(2):1046.

DOI: https://doi.org/10.26420/jbacteriolmycol.2017.1046

9. Limmathurotsakul D, Wuthiekanun V, Amornchai P, Wongsuwan G, Day NP, Peacock SJ. Effectiveness of a simplified method for isolation of Burkholderia pseudomallei from soil. Appl Environ Microbiol. 2012;78(3):876-7. DOI: https://doi.org/10.1128/AEM.07039-11

10. Limmathurotsakul D, Golding N, Dance DA, Messina JP, Pigott DM, Moyes CL, et.al. Predicted global distribution of Burkholderia pseudomallei and burden of melioidosis. Nature microbiology, 2016; 1(1),15008.

DOI: https://doi.org/10.1038/nmicrobiol.2015.8

11. Hoffmaster AR, AuCoin D, Baccam P. Melioidosis diagnostic workshop. Emerg Infect Dis. 2015;21(2);141045. DOI: https://doi.org/10.3201/eid2102.141045 